\title{
A POSITIVE FUNCTION WITH VANISHING LEBESGUE INTEGRAL IN ZERMELO-FRAENKEL SET THEORY
}

\author{
VLADIMIR KANOVEI AND MIKHAIL G. KATZ
}

\begin{abstract}
Can a positive function on $\mathbb{R}$ have zero Lebesgue integral? It depends on how much choice one has.

Keywords: Lebesgue integral; Zermelo-Fraenkel theory; FefermanLevy model. 03E25; 28A25
\end{abstract}

\section{Contents}

1. Introduction

2. On Lebesgue measure in the Feferman-Levy model

3. A positive function with zero Lebesgue integral in FL

4. Violation of Fubini

Acknowledgments

References

\section{INTRODUCTION}

It is known that the proposition

(*) the Lebesgue measure is countably additive

is a weak form of the axiom of choice, i.e., it cannot be proved in Zermelo-Fraenkel set theory ZF and it does not imply the axiom of choice AC. The Feferman-Levy model (model $\mathcal{M} 9$ in the monograph [5]) witnesses the failure of $(*)$. Thus, if one wishes to work in a $\mathrm{ZF}$ environment where $\mathrm{AC}$ fails, one has to give up countable additivity. We show that, relative to a definition of Lebesgue measure suitable for working in $\mathcal{M}$, the Fubini theorem and the assertion that "a positive real-valued function on $[0,1]$ has positive integral" both fail in $\mathcal{M} 9$.

Based on the typical working mathematician's intuitions of $\mathbb{R}$, it seems reasonable to expect that, on the one hand,

(1) a function which is positive everywhere could not have a vanishing Lebesgue integral; and, on the other, 
(2) this fact should admit a reasonably constructive proof, such as one based on the traditional Zermelo-Fraenkel set theory (ZF).

Curiously, while item (11) is correct as stated, item (2) is incorrect and in fact there exist models of ZF admitting positive functions with vanishing Lebesgue integral.

We start with the following geometric observation. In a standard Möbius band $M$, every interval orthogonal to the central circle of $M$ is isometric to the segment $(-\epsilon, \epsilon)$, but it is impossible to choose such isometries simultaneously for all such intervals on $M$ in a continuous fashion, for that would imply that $M$ is a cylinder (rather than a Möbius band).

To explain a similar phenomenon in a set-theoretic context, we first introduce a distinction between a countable set $A$, for which there exists a one-to-one correspondence between $A$ and $\mathbb{N}$, and a counted set $B$, for which a specific identification $\iota_{B}: B \rightarrow \mathbb{N}$ has been chosen; thus $B$ is shorthand for the pair $\left(B, \iota_{B}\right)$.

A countable family $\left\{B_{\alpha}\right\}_{\alpha \in \mathbb{N}}$ of (disjoint) counted sets $B_{\alpha}$ is immediately identifiable with $\mathbb{N} \times \mathbb{N}$. Therefore the union $\cup_{\alpha \in \mathbb{N}} B_{\alpha}$ is countable by a snaking argument in $\mathbb{N} \times \mathbb{N}$ familiar to each freshman, in the context of enumerating $\mathbb{Q}$.

The snaking argument does not a priori work in ZF for a countable family $\left\{A_{\alpha}\right\}_{\alpha \in \mathbb{N}}$ of (disjoint) countable sets $A_{\alpha}$, because constructing an identification with $\mathbb{N} \times \mathbb{N}$ requires choosing a particular identification of $A_{\alpha}$ with $\mathbb{N}$ simultaneously for all $\alpha$. This procedure requires the axiom of countable choice (ACC).

This might seem like a limitation of the particular proof of countability that one might be able to overcome by a more judicious procedure avoiding ACC. However, it turns out that the obstruction is genuine.

Cohen's work on the continuum hypothesis soon led to a model of ZF, called the Feferman-Levy (FL) model [2], where the real number line $\mathbb{R}$ is a countable disjoint union of countable (but not counted) sets, whereas of course $\mathbb{R}$ itself is uncountable by the classical diagonal argument of Cantor (which does not rely on AC); see [1, chapter IV, section 4]. The Lebesgue measure is not $\sigma$-additive in FL.

These phenomena do not contradict the categoricity of $\mathbb{R}$ (namely, its characterisation as the unique complete ordered field) since, as is well known, the concept of categoricity is dependent on the background model of set theory.

\section{On Lebesgue measure in the Feferman-LeVy MOdeL}

A crucial property of the Feferman-Levy model is the following: 
(FL) The unit interval $[0,1]$ is equal to a countable union $[0,1]=\bigcup_{n} C_{n}$ of countable sets $C_{n} \subseteq[0,1]$.

Due to property (FL), not every definition of the Lebesgue measure is appropriate in this model. In particular, any definition that explicitly involves the algebra of Borel sets and countable additivity, as e.g., in Halmos [4], is immediately ruled out as incompatible with property (FL).11 Therefore we begin with an explicit definition of the Lebesgue measure suitable for working with in any ZF environment, including the choiceless FL model. A set $X \subseteq \mathbb{R}$ is bounded if $X \subseteq[-c, c]$ for some finite $c<+\infty$. We will consider the measure with respect to bounded sets only. The length len $(I)$ of any open or closed real interval $(a, b)$ or $[a, b]$ is equal to $b-a$.

Definition 2.1 (see [10], 1.2.2, or [8] in slightly different terms). The outer measure $m^{*}(X)$ of a bounded set $X \subseteq \mathbb{R}$ is equal to the infimum of $\sum_{n} \operatorname{len}\left(I_{n}\right)$ over all coverings $X \subseteq \bigcup_{n} I_{n}$ of $X$ by countably many open intervals $I_{n}$.

A bounded set $X \subseteq \mathbb{R}$ is Lebesgue measurable (LM for short) if for every $\varepsilon>0$, there exists an open set $U$ containing $X$ such that $m^{*}(U \backslash X)<\varepsilon$. If $X$ is Lebesgue measurable, then $m(X):=m^{*}(X)$ is the Lebesgue measure of $X$.

The same definition of $m^{*}$ and $m$ works in any $n$-dimensional Euclidean space $\mathbb{R}^{n}, n \geq 2$, with intervals replaced by boxes, i.e., Cartesian products of $n$-many finite real intervals.

The following properties of the measure are provable in $\mathrm{ZF}$ and hence true in the FL model.

(I) every interval $I=[a, b]$ or $(a, b)$ is LM and satisfies $m(I)=$ $\operatorname{len}(I)$;

(II) $m$ is finitely additive;

(III) every finite or countable bounded set $X \subseteq \mathbb{R}$ is LM and satisfies $m(X)=0$.

It follows by (FL) that $m$ is not countably additive in the FL model.

Definition 2.2. A real function $f: I \rightarrow \mathbb{R}$ defined on a bounded real interval $I$ is Lebesgue measurable (LM) iff each superlevel set $\{x \in$ $I: f(x)>y\}$ is LM. In such case the auxiliary function $g(y)=m(\{x \in$ $I: f(x)>y\})$ is monotone and has a well-defined Riemann integral. The Lebesgue integral $\int_{I} f d x$ is defined to be equal to the Riemann integral $\int_{0}^{\infty} g(y) d y$.

\footnotetext{
${ }^{1}$ However see Fremlin [3] for a hard roundabout in terms of codable countable unions, which enables one to salvage some countable additivity even in fully non$\mathrm{AC}$ environments.
} 


\section{A positive Function With Zero Lebesgue integral in FL}

D. Fremlin [3] presents a careful development of Lebesgue integration in a ZF context and shows that it satisfies a fundamental theorem of calculus. Note that various definitions that are equivalent in a ZFC context may become inequivalent over $\mathrm{ZF}$.

We show that using the traditional definitions presented in Section 2 the Lebesgue integral of our positive function is zero.

Theorem 3.1 (in ZF). Suppose that $[0,1]=\bigcup_{n \in \mathbb{N}} A_{n}$, where each $A_{n}$ is countable (as e.g., in FL). Then there is a positive real function on $[0,1]$ with zero Lebesgue integral.

Proof. Suppose $[0,1]=\bigcup_{n \in \mathbb{N}} A_{n}$ where each $A_{n}$ is countable. We will rely on Definition 2.2 of the Lebesgue integral. Consider the function $f$ equal to $\frac{1}{n}$ on the $n$-th countable set $A_{n}, n=1,2,3, \ldots$, or in formulas $f L_{A_{n}}=\frac{1}{n}$; alternatively, $f=\sum_{n} \frac{1}{n} \chi_{A_{n}}$. Then the auxiliary function $g$ is identically zero. Indeed, its superlevel sets are finite unions of the form $A_{1} \cup A_{2} \cup \cdots \cup A_{n}$, hence, countable sets.2 Therefore the Riemann integral of $g$ is zero, and hence the Lebesgue integral of the given positive function $f$ is also zero.

The definition of Lebesgue integral in terms of simple functions gives the same result. For our function $f(x)$ which equals $\frac{1}{n}$ when $x \in A_{n}$, every simple function $s(x) \geq 0$ dominated by $f$ will be nonzero at most at countably many points. Therefore the Riemann integral of $s(x)$ is zero. Therefore the Lebesgue integral of $f$, which is the supremum of the integrals of the simple functions, is also zero. The infimum of the integrals of simple functions that dominate $f$ is similarly 0 .

Indeed, given a small $\epsilon=\frac{1}{m}$ where $m \geq 1$, we define $s$ by setting $s(x)=f(x)=\frac{1}{n}$ whenever $x$ belongs to $A_{n}$ and $n \leq m$, and $s(x)=\frac{1}{m}$ whenever $x$ belongs to $A_{n}$ and $n>m$. Then $s$ is a simple function whose Lebesgue integral by definition is equal to $I_{1}+I_{2}+\cdots+I_{m}+I$, where each $I_{n}=\frac{1}{n} \cdot \mu\left(A_{n}\right)$ is equal to 0 since $A_{n}$ is countable, while $I=\frac{1}{m} \mu\left(\bigcup_{n>m} A_{n}\right) \leq \frac{1}{m} \mu([0,1])=\frac{1}{m}$, so altogether $\int_{0}^{1} s d \mu \leq \frac{1}{m}$, as required. Here $\mu$ denotes the Lebesgue measure on $[0,1]$, and the axiom of choice is not used in this elementary argument.

\footnotetext{
${ }^{2}$ Note that there is no need for the axiom of choice to prove that a finite union of countable sets is itself countable, and that a countable set has Lebesgue measure 0; see [3]. Furthermore, $f$ is a Lebesgue-measurable function; indeed the $f$-preimage of any interval $(a,+\infty)$ is either the entire interval $[0,1]$ if $a \leq 0$, or a finite union of sets $A_{n}$ otherwise, in which case it is a countable set.
} 


\section{Violation of Fubini}

The original and best known form of the Fubini Theorem claims that under certain conditions two iterated integrals coincide. However there is another formulation of the result, which does not directly involve integration. This form, useful in the context of Lebesgue measure theory, is similar to the Kuratowski-Ulam theorem (see [7, 8.41]) related to Baire category rather than measure. Namely we have the following; cf. [3, exercise 565X(e), p. 221].

Proposition 4.1 (Fubini). Assume that $P \subseteq \mathbb{R}^{2}$ is a Lebesgue measurable set. Then $P$ is null if and only if the set $X$ of all reals $x$ such that "the cross-section $P_{x}=\{y:(x, y) \in P\}$ is non-null", is null as well.

Theorem 4.2 (in ZF). In the hypotheses of Theorem 3.1, Proposition 4.1 fails.

Proof. Consider a decomposition $I=\bigcup_{n} A_{n}$ of the unit interval $I=$ $[0,1]$ where each $A_{n}$ is countable. Now let $f: I \rightarrow \mathbb{R}$ be the function defined as in the proof of Theorem 3.1 . Let $P$ be the set of all pairs $(x, y)$ such that $0<y<f(x)$. We claim that $P$ is a null set. Indeed, given $\epsilon=\frac{1}{n}$, we have to cover $P$ by an open planar set $U$ of measure $<\frac{2}{n}$. We let $P=P^{\prime} \cup P^{\prime \prime}$, where $P^{\prime}=\left\{(x, y) \in P: x \in \bigcup_{k<n} A_{k}\right\}$ and $P^{\prime \prime}=$ $\left\{(x, y) \in P: x \in \bigcup_{k \geq n} A_{k}\right\}$. Note that if $x \in A_{k}, k \geq n$, then $f(x) \leq \frac{1}{n}$, therefore $P^{\prime \prime}$ is covered by the open rectangle $U^{\prime \prime}=(0,1) \times\left(0, \frac{1}{n}\right)$ of measure $\frac{1}{n}$.

On the other hand, the projection of $P^{\prime}$ to the horizontal axis is equal to the countable set $I^{\prime}=\bigcup_{k<n} A_{k}$. Therefore $I^{\prime}$ is a null set, and we can cover it by an open set $G$ of measure $<\frac{1}{n}$. Then $P^{\prime}$ is covered by $U^{\prime}=G \times[0,1]$, an open set of planar measure $<\frac{1}{n}$.

Finally, the union $U=U^{\prime} \cup U^{\prime \prime}$ is an open planar set of measure $<\frac{2}{n}$ which covers $P$, as required. Thus $P$ is a null set.

On the other hand, if $x \in I$ then the vertical cross-section $P_{x}$ defined by $P_{x}=\{y:(x, y) \in P\}$ is a non-empty open interval, hence, a non-null set. It follows that the set $X$ as in Proposition 4.1 is equal to $I$, and hence is non-null. Thus $P$ violates Proposition 4.1, as required.

There are various definitions of integration, and the relations among them and the Lebesgue measure, to which mathematicians are accustomed, sometimes depend on the axiom of choice (AC). Accordingly, if $\mathrm{AC}$ fails then such relationships may fail as well. In particular, in 
the FL model, where even countable choice is not available, an elementary standard property of integration fails as well, as we demonstrate in this note.

\section{ACKNOWLEDGMENTS}

We are grateful to the referees for a number of helpful suggestions. V. Kanovei was supported in part by the RFBR grant number 17-0100705. M. Katz was partially funded by the Israel Science Foundation grant number 1517/12.

\section{REFERENCES}

[1] P. Cohen, Set theory and the continuum hypothesis. Undergraduate Texts in Mathematics. NY - Amst.: W.A. Benjamin, 1966.

[2] S. Feferman and A. Levy, Independence results in set theory by Cohen's method II, Notices Amer. Math. Soc., 10 (1963), 593.

[3] D. H. Fremlin, Measure theory. Vol. 5. Set-theoretic Measure Theory. Part II, Torres Fremlin, Colchester, 2008.

[4] P. Halmos, Measure Theory, Graduate Texts in Mathematics vol. 18, Springer, New York, 1976.

[5] P. Howard and J. E. Rubin, Consequences of the axiom of choice. With 1 IBMPC floppy disk (3.5 inch; WD), Mathematical Surveys and Monographs, 59. American Mathematical Society, Providence, RI, 1998.

[6] Th. Jech, The axiom of choice, Studies in Logic and the Foundations of Mathematics, Vol. 75, North-Holland, 1973.

[7] A. Kechris, Classical descriptive set theory, Graduate Texts in Mathematics, 156. Springer-Verlag, New York, 1995.

[8] A. Kolmogorov and S. Fomin, Elements of the theory of functions and functional analysis. Vol. 2: Measure. The Lebesgue integral. Hilbert space, Translated from the first (1960) Russian edition by Hyman Kamel and Horace Komm, Graylock Press, Albany, NY, 1961.

[9] K. Kunen, Set theory, Studies in Logic, 34. London: College Publications, 2011; Revised edition 2013.

[10] T. Tao, An introduction to measure theory, Graduate Studies in Mathematics, 126. American Mathematical Society, Providence, RI, 2011.

V. Kanovei, Laboratory 6, IPPI RAN, Bolshoy Karetny Per. 19, Build. 1, Moscow 127051, Russia; And MitT, 9в9 Obrazcova St, 127994 Moscow, Russia

E-mail address: kanovei@googlemail.com

M. Katz, Department of Mathematics, Bar Ilan University, Ramat GAN 5290002 ISRAEL

E-mail address: katzmik@macs.biu.ac.il 